Symétrie spatiale et temporelle d'une enclave : la Prusse-Orientale (1919-1939) et Kaliningrad (1992-2004)

Spatial and temporal symmetry of an enclave: East Prussia (1929-1939) and Kaliningrad (1992-2004)

Räumliche und zeitliche Symmetrie einer Enklave : Ostpreußen (1919-1939) und Kaliningrad (1992-2004)

\title{
Frank Tetart
}

\section{(2) OpenEdition}

\section{Journals}

Édition électronique

URL : http://journals.openedition.org/rge/207

ISSN : 2108-6478

Éditeur

Association des géographes de l'Est

Édition imprimée

Date de publication : 1 avril 2007

ISSN : 0035-3213

Référence électronique

Frank Tetart, «Symétrie spatiale et temporelle d'une enclave : la Prusse-Orientale (1919-1939) et Kaliningrad (1992-2004) », Revue Géographique de l'Est [En ligne], vol. 47 / 2 | 2007, mis en ligne le 22 décembre 2009, consulté le 07 septembre 2020. URL : http://journals.openedition.org/rge/207

Ce document a été généré automatiquement le 7 septembre 2020

Tous droits réservés 


\section{Symétrie spatiale et temporelle d'une enclave : la Prusse-Orientale (1919-1939) et Kaliningrad (1992-2004)}

Spatial and temporal symmetry of an enclave: East Prussia (1929-1939) and Kaliningrad (1992-2004)

Räumliche und zeitliche Symmetrie einer Enklave : Ostpreußen (1919-1939) und Kaliningrad (1992-2004)

Frank Tetart

\section{NOTE DE L'ÉDITEUR}

Article reçu le 20 novembre 2006, accepté le 15 juin 2007

\section{Kaliningrad/Prusse-Orientale comme construction territoriale d'une symétrie de l'enclavement}

Enclavée entre la Pologne au Sud et la Lituanie au Nord et à l'Est (carte 1), la région russe de Kaliningrad n'a plus depuis 1991 aucun lien de continuité avec le reste de la Fédération de Russie, puisque sa frontière avec la Lituanie qui n'était à l'époque soviétique qu'une limite administrative interne, est devenue une frontière internationale. Il est désormais nécessaire de franchir trois frontières pour rejoindre, Pskov, la ville russe la plus proche, située à 600 km de Kaliningrad. 
Carte 1 : Kaliningrad, une enclave russe sur la Baltique.

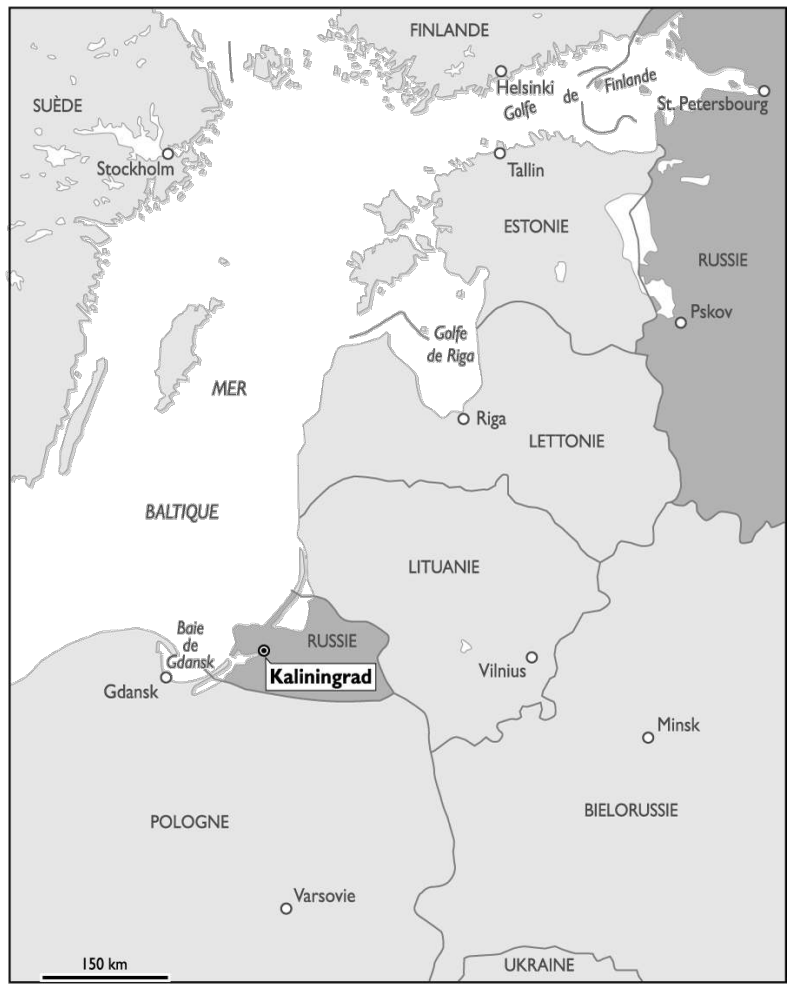

\section{A. Une exclave russe}

2 Pour Moscou, cette discontinuité territoriale est considérée comme une contrainte, autant pour la séparation que pour l'isolement qu'elle implique pour Kaliningrad vis-àvis du reste de la Russie. En dépit de sa façade maritime sur la Baltique, la région est dès lors représentée comme une enclave ou plutôt comme une exclave. Ce néologisme largement utilisé en Russie par la presse et parmi les spécialistes, permet de qualifier à la fois l'enclavement de la région russe dans l'espace Baltique et sa situation « insulaire » par rapport au reste du territoire russe. D'emblée, l'enclavement prend une valeur négative, car il soulève la question de la circulation des personnes, des échanges, du commerce et par conséquent de la viabilité d'un territoire de faible étendu et très dépendant de sa métropole. Or, ces problèmes se sont fortement accentués à la fin des années 1990 avec les perspectives de l'élargissement de l'Union européenne et de l'OTAN aux voisins de Kaliningrad, que Moscou regarde avec hostilité.

Aussi, à partir de 2002, Kaliningrad se retrouve-t-elle au cœur de l'actualité des relations russo-européennes. La politique de Moscou vis-à-vis de Kaliningrad, une région jusque-là d'intérêt purement stratégique - se focalise sur la question des visas qui, en application des règles de l'espace Schengen, sont exigés des habitants de Kaliningrad en transit vers la Russie à travers la Lituanie. Les habitants de Kaliningrad bénéficiaient depuis la fin de l'URSS d'un régime de faveur, leur permettant de franchir les frontières de cet Etat sans visa, sur simple présentation de leur passeport intérieur comme à l'époque soviétique, et d'y séjourner pendant 30 jours. Deux représentations se font alors face : sécuritaire côté européen, souverainiste côté russe [Tétart, 2005]. Le pouvoir russe craint que la mise en place de visas ne favorise des tendances séparatistes 
dans la région. Bruxelles, pour sa part, cherche à protéger le territoire européen, les frontières entre l'enclave et la Pologne d'une part, et la Lituanie d'autre part, devenant des frontières extérieures de l'Union. La question de Kaliningrad exacerbe les tensions entre la Russie et l'Union européenne et suscite des craintes chez les nouveaux entrants : la Pologne et avant tout la Lituanie, qui sert au transit.

Ce contexte d'enclavement rappelle celui issu de la Première Guerre mondiale, où ce même territoire - qui était alors la province allemande de Prusse-Orientale - s'était retrouvé séparé par le corridor de Dantzig du reste de sa "mère-patrie » l'Allemagne, cette fois située sur son flanc Ouest, suite à la perte de territoires au profit de la Pologne reconstituée en 1919 (Voir Carte 2).

Carte 2 : La Prusse-Orientale, séparée de l'Allemagne par le Traité de Versailles.

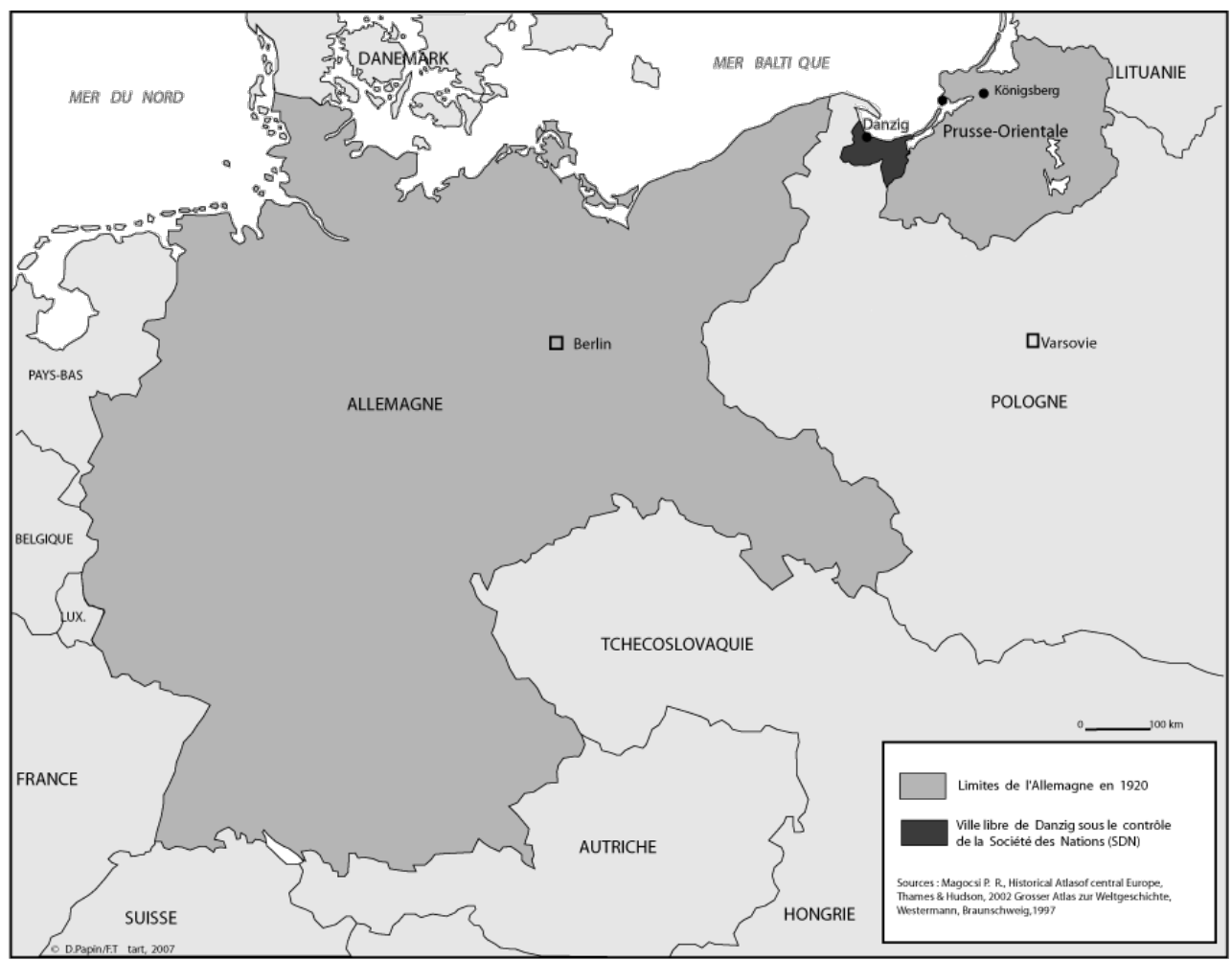

\section{B. La Prusse-Orientale, un enclavement imposé à l'Allemagne}

Dès janvier 1918, le président américain Wilson avait déclaré, en évoquant le futur Etat polonais, qu'on devait lui garantir «un libre et sûr accès à la mer $»^{2}$. Comme les inconvénients que pouvaient présenter les nouvelles frontières pour la PrusseOrientale étaient négligeables aux yeux des Alliés, comparés à ceux que tout autre arrangement causerait à la Pologne ${ }^{3}$, on amputa l'Allemagne de la Pomérélie ${ }^{4}$ et de Dantzig, qui fut érigé en ville libre par le Traité de Versailles, sous contrôle de la Société des Nations. La Pologne obtint ainsi $146 \mathrm{~km}$ linéaires de côtes. Conformément au Traité de Versailles, la Province de Prusse-Orientale se retrouva enclavée entre le nouvel Etat polonais reconstitué, la ville libre de Dantzig, et la Lituanie nouvellement indépendante au Nord. Elle n'avait plus de contact direct avec les provinces allemandes, d'ailleurs comme avant les partages de la Pologne de la fin du XVIII ${ }^{\mathrm{e}}$ siècle. Une configuration qui remontait à la Paix de Torun de 1466, où le Duché de Prusse 
formé au début du XIII siècle par les Chevaliers Teutoniques avait perdu ces territoires situés entre les fleuves Oder et Vistule au détriment de la Pologne, le coupant du reste des territoires de langue et de civilisation allemandes, alors rassemblés dans le Saint Empire Romain Germanique.

6 Aussi, après la Première Guerre mondiale, les pertes territoriales de l'Allemagne sontelles considérées comme "une répétition de l'injustice subie en 1466 ». Ce qu'on appelait alors «le combat pour la Vistule » apparait comme "une constante des relations entre l'Allemagne et la Pologne ", si bien que, dans les deux pays, cette rivalité de territoire est alors perçue - selon les termes de Boockmann [1992, p. 255] - comme une "prédisposition géopolitique» du voisin, qui ne peut qu'inexorablement se répéter: côté allemand, pour réaliser la formation d'un territoire continu et, côté polonais, pour obtenir un accès à la mer ${ }^{5}$.

7 La reconstitution de la Pologne en 1919, en mettant fin à la continuité territoriale de l'Allemagne transforme la Province de Prusse-Orientale en une enclave séparée du reste de l'Allemagne par le corridor de Dantzig. Dès lors, l'enclavement apparaît pour l'Allemagne comme un handicap, qu'il est nécessaire de combattre au nom d'un « droit naturel ", dans une vision nationaliste, pour mettre fin à cette discontinuité. Il se révèle être une source de tensions et de conflits entre l'Allemagne et la Pologne - avant d'être le prétexte au déclenchement de la Seconde Guerre mondiale.

\section{Une rupture politique à l'origine de l'enclavement}

Dans une symétrie spatiale et temporelle, la Prusse-Orientale apparaît donc comme le double de Kaliningrad. Dans les deux cas, la genèse de l'enclavement naît d'une rupture politique, que l'on peut même qualifier de géopolitique, tant elle est liée à un changement de rapport de forces: la défaite allemande qui conduit au repli des Empires (allemand, russe et autrichien) en Europe centrale, en ce qui concerne la Prusse-Orientale, et la fin de la Seconde Guerre mondiale et la dissolution de l'Union soviétique, dans le cas de Kaliningrad.

9 Ces deux bouleversements majeurs des rapports de puissance en Europe sont à l'origine du statut particulier de Kaliningrad. Les accords de Yalta (février 1945) et de Potsdam (juillet-août 1945) attribuèrent la partie nord de la Prusse-Orientale allemande, avec la ville de Königsberg, aux Soviétiques ${ }^{6}$, la partie sud revenant à la Pologne. En devenant en 1946 une entité territoriale de l'Union soviétique, directement rattachée à la République Socialiste Soviétique Fédérative de Russie (RSSFR), le sort de la région fut dès lors irrémédiablement scellé à celui de la Russie, au sein de l'URSS. La région fut rebaptisée Kaliningrad, tout comme sa capitale administrative Königsberg, en l'honneur de Michaël Kalinine, président du Soviet Suprême décédé en juillet 1946. Les populations allemandes qui n'avaient pas fui en 1945 devant l'avancée de l'Armée rouge furent expulsées en totalité vers l'Allemagne à l'automne 1948, après avoir servi de main-d'œuvre au démarrage de l'économie locale et surtout à l'approvisionnement en produits agricoles de l'Armée rouge et des experts soviétiques, dans l'attente de l'installation de populations soviétiques. Celles-ci, espérant échapper à la misère de l'après-guerre, avaient commencé à affluer en 1946 de l'ensemble de l'Union soviétique, en particulier de sa partie occidentale très touchée par la guerre et l'occupation allemande ${ }^{7}$. 
10 Alors que l'enclavement de la Prusse-Orientale avait causé une émigration limitée de la population allemande - dans la mesure où elle était, selon Jacques Ancel [1933], avant tout la résultante de problèmes liés à la structure économique de la province -, l'annexion soviétique mit fin à 700 ans de présence allemande et à sa transformation en un avant-poste militaire, véritable « enclave » à l'intérieur de l'URSS.

\section{Gérer l'enclavement : approches symétriques entre Kaliningrad et son double, la Prusse-Orientale}

Pour toute enclave, la question vitale est d'abord celle de son accessibilité [Nies, 2003]. Dans les contextes de séparation de Kaliningrad et de son double la Prusse-Orientale, comment gérer la discontinuité territoriale et permettre les liaisons et les échanges avec le reste du pays ? Le problème de la discontinuité territoriale soulève en priorité la gestion du transit avec l'Etat enclavant et la question des transports - au sens large nécessaires aux déplacements, aux flux commerciaux et à l'approvisionnement énergétique.

\section{A. Le cadre du transit}

Deux articles du Traité de Versailles définissent le cadre du transit par le territoire polonais des communications de la Prusse-Orientale vers l'Allemagne. Dans l'article 89 est établi le principe de la «liberté de transit » pour le trafic entre l'Allemagne et la Prusse-Orientale, via les seules Voïvodies de Poméranie et de Posnanie. Cet article exempt les marchandises en transit de tout droit de douane. Il est complété par l'article 98, qui prévoit l'établissement d'une convention entre l'Allemagne et la Pologne pour régler le problème des communications par voie ferrée, télégraphe et téléphone, entre l'Allemagne et la Prusse-Orientale à travers la Pologne et le territoire de la Ville Libre de Dantzig d'une part, et entre la Pologne et la Ville libre de Dantzig, à travers la Prusse-Orientale le long de la rive droite de la Vistule, d'autre part ${ }^{8}$. Cette convention «sur la liberté du transit entre la Prusse Orientale et le reste de l'Allemagne » est signée à Paris le 21 avril 1921 et entre en vigueur le $1^{\text {er }}$ juin 1922. D'après un rapport du Ministère des Affaires étrangères allemand ${ }^{9}$, ce sont les besoins économiques et les moyens de communications de l'Allemagne qui décideront de l'importance de l'ampleur que prendra le transit. Néanmoins, deux restrictions sont imposées : d'abord, en cas de guerre, les dispositions de la convention cesseront ipso jure; ensuite, le transit étant soumis aux lois polonaises, notamment pour la sécurité publique, il ne jouit pas du privilège de l'exterritorialité. Ainsi, en cas de crimes ou délits graves, les autorités polonaises peuvent procéder à des arrestations. 
Carte 3 : Le transit allemand via le corridor de Dantzig par voie de chemin de fer.

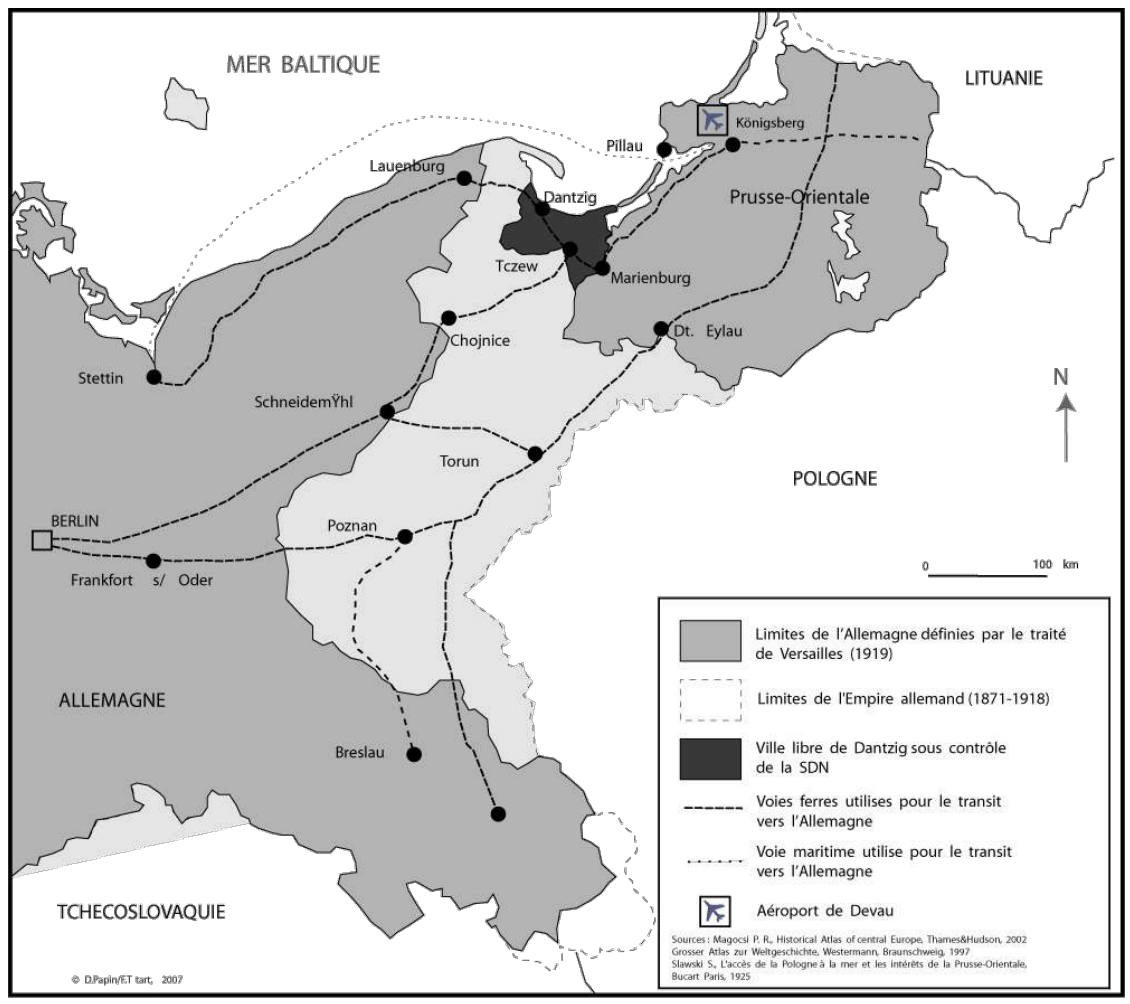

Concrètement, cette convention prévoit un transit par voie ferrée, dit "privilégié », et un transit ordinaire. Le transit privilégié s'effectue sur huit lignes déterminées ${ }^{10}$ sans aucune formalité de passeport et de douanes pour les personnes et marchandises. En cas de guerre, néanmoins, les citoyens allemands devront être munis de cartes d'identité et ceux des autres Etats d'un passeport portant le visa polonais. Il reste cependant défendu aux voyageurs de descendre du train en cours de voyage et pendant l'arrêt dans les gares, de même que de passer et recevoir des objets par les portières. En ce qui concerne le transit ordinaire, qui peut se faire sur toutes les lignes de chemin de fer de Poméranie et de Posnanie, les voyageurs de nationalité allemande doivent être munis d'une carte d'identité portant le visa polonais. La convention a également prévu un train par semaine pour le transport des soldats et permissionnaires, et un autre pour le transport du matériel militaire.

En 1929, 24 trains de transit circulent chaque jour. Ils ont transporté, durant cette même année, 458800 voyageurs, selon le géographe français Jacques Ancel, qui a pu par lui-même se rendre compte de la facilité avec laquelle tout voyageur peut franchir la frontière [Ancel, 1932]. Il dénonce donc les procédés de la Geopolitik qui représente sur des cartes le trafic ferroviaire en 1928 en interrompant les parcours dans le corridor, comme s'il n'y avait pas un seul train qui passait dans le " couloir »! De fait, dès l'année 1924, entre la Prusse-Orientale et l'Allemagne, le transit par train représente $68 \% \mathrm{du}$ trafic voyageurs pour seulement $32 \%$ par mer (ligne Königsberg-Stettin) [Slawski, 1925, p. 126]. Pour les échanges de marchandises en vrac, le trafic via la Pologne double même entre 1913 et 1928, passant de 2,3 millions de tonnes transportées à 4,5 millions [Ancel, 1932]. Le transit tant des voyageurs que des marchandises se réalise donc sans entraves. Par ces arrangements juridiques, les communications entre la PrusseOrientale et l'Allemagne se trouvent assurées par chemin de fer de manière régulière, 
les lignes de transit constituant pour l'Allemagne "comme un territoire roulant" [Slawski, 1925, p. I-XII].

Dans le cas de Kaliningrad, la question de l'accessibilité est assez paradoxale, puisque le territoire, du fait de sa fonction d'avant-poste militaire (siège de la Flotte de la Baltique) pendant la Guerre Froide, est resté fermé aux étrangers et à la grande majorité des Soviétiques jusqu'à la fin de l'URSS. L'indépendance de la Lituanie qui le coupe de la Russie oblige certes Moscou à la négociation ; mais le libre transit des véhicules et des trains, sur simple présentation du passeport soviétique, est en réalité quasiment échangé contre le retrait de l'Armée rouge, avec le nouvel Etat indépendant. On comprend dès lors les réticences de Moscou à accepter la remise en cause de ce régime de faveur, du fait de l'élargissement européen. Néanmoins, après de nombreuses tergiversations et une période de tensions avec l'Union européenne, le gouvernement fédéral russe signe en novembre 2002 avec Bruxelles, un accord sur la mise en place d'un document de transit facilitant le passage des ressortissants russes en direction et en provenance de Kaliningrad. Un compromis "de substance pour Moscou » et «de forme pour Bruxelles », selon l'analyste Dov Lynch [Lynch, 2003 (2)], puisque Bruxelles par pragmatisme évite d'utiliser le terme de "visa ", pour désormais parler de « document de transit facilité » (FTD- facilitated transit document), alors qu'il s'agit d'une version simplifiée et gratuite du régime Schengen de visas ${ }^{11}$. Il permet à la Russie de ne pas apparaître dans une posture de faiblesse vis-à-vis de l'Union européenne, et qui plus est, de la Lituanie. Symboliquement, du fait de l'histoire soviétique, dont les Russes se sentent les héritiers, la fin de la continuité territoriale par la mise en place de visas était peut-être ressentie comme une "insulte», car émanant de peuples qu'ils avaient longtemps dominés (Lituaniens et Polonais). De son côté, la Lituanie ne voulait en aucun cas devenir un Etat-membre de l'Union de "seconde zone", ni se retrouver dans une position de vulnérabilité vis-à-vis de la Russie, à cause de dispositions particulières pour les habitants de Kaliningrad, dérogeant ainsi à l'acquis communautaire.

Dans le cas de la Prusse-Orientale, comme de Kaliningrad, le cadre juridique du transit parvient à concilier liberté de circulation et respect de l'exercice de la souveraineté des Etats enclavants (Pologne, Lituanie), sans entraver le quotidien des populations de l'enclave. D'autant que les règles de transit sont complétées par des mesures concrètes pour pallier et gérer les conséquences de sa séparation d'avec le reste de sa patrie (Russie pour Kaliningrad et Allemagne pour la Prusse-Orientale) et de l'isolement induit par cette nouvelle configuration.

\section{B. Mesures en faveur des infrastructures}

Ces mesures sont de deux ordres, économique et politique. Elles sont soit prises conjointement entre les autorités locales et le gouvernement central, soit elles relèvent d'une intervention directe de l'Etat central sous forme d'aménagements ou de subventions, notamment en faveur des transports.

Ainsi, à partir des années 1920, plusieurs aménagements sont réalisés en PrusseOrientale. En 1919, un aéroport international est construit à Devau, dans la partie Est de Königsberg. Il s'agit du premier aérodrome civil d'Allemagne et il permet à la PrusseOrientale de s'intégrer dans le réseau aérien européen naissant. Devau est le point de départ de lignes reliant Königsberg à Berlin et Dantzig, ainsi que Smolensk et Moscou 
(en Russie), Memel, Riga et Reval ${ }^{12}$ (dans les pays Baltes).Au printemps 1920, est mis en place le service maritime de Prusse-Orientale entre Pillau et Swinemünde (au Nord de Stettin), afin de créer une liaison indépendante entre la Prusse-Orientale et le reste du pays, sans transit terrestre via la Pologne. Cette liaison maritime est étendue quelques années plus tard à Travemünde (port au Nord de Lübeck) et à Helsinki. Enfin, une gare centrale (Hauptbahnhof) est inaugurée en 1929 à Königsberg, pour desservir le sud et l'ouest de la Prusse-Orientale et faciliter le trafic ferroviaire vers le reste de l'Allemagne. Enfin, l'université de Königsberg reçoit une importante dotation en fournitures et en 1924 est installé à Königsberg l'émetteur radio de la Marche de l'Est (Ostmarkenrundfunk AG).

Comme pour la Prusse-Orientale, la voie terrestre n'est pas la seule voie de transport pour se rendre à Kaliningrad depuis la Russie ou de Kaliningrad vers le reste du pays. La région dispose d'un aéroport à Khrabrovo, à $40 \mathrm{~km}$ au Nord de la ville de Kaliningrad et de plusieurs ports, grâce à son ouverture maritime sur la Baltique (Voir carte 4). Aussi la deuxième réponse apportée pour gérer l'isolement et la séparation du reste du territoire russe est-elle celle du pragmatisme de la part du gouvernement russe. En effet, quelques jours avant la mise en place du nouveau régime des visas en Lituanie le $1^{\text {er }}$ juillet 2003, le gouvernement Poutine annonce la baisse des tarifs aériens, les alignant sur ceux du train. En l'espace d'une semaine, le nombre de voyageurs sur cette ligne augmente de $800 \%$. Au total, la compagnie transporte en 2004 sur cette ligne 280 000 passagers ${ }^{13}$. En décidant de subventionner une partie du prix des voyages en avion, les autorités ont fait le choix de l'adaptation, après la confrontation avec l'Union européenne. Ce qui souligne, d'une part, le désintérêt marqué du Centre vis-à-vis de Kaliningrad tout au long de la décennie 1990 et laisse supposer, d'autre part, que Kaliningrad a été un instrument utilisé « au maximum » de sa capacité, pour s'opposer de façon indirecte à l'élargissement européen et de l'OTAN. D'ailleurs, Viacheslav Nikonov, président de la FondationPolitika, déclare en juin 2002 dans une interview au journal Strana : «il est erroné de décrire Kaliningrad comme un territoire encerclé, la Russie devrait plutôt concentrer ses efforts sur l'amélioration des communications par ferry ou par avion entre la région et le reste du pays ${ }^{14}{ } »-$ sous-entendant que la Russie est confrontée à Kaliningrad à des problèmes techniques plutôt que purement politiques. 
Carte 4 : Les principales voies de communication de Kaliningrad.

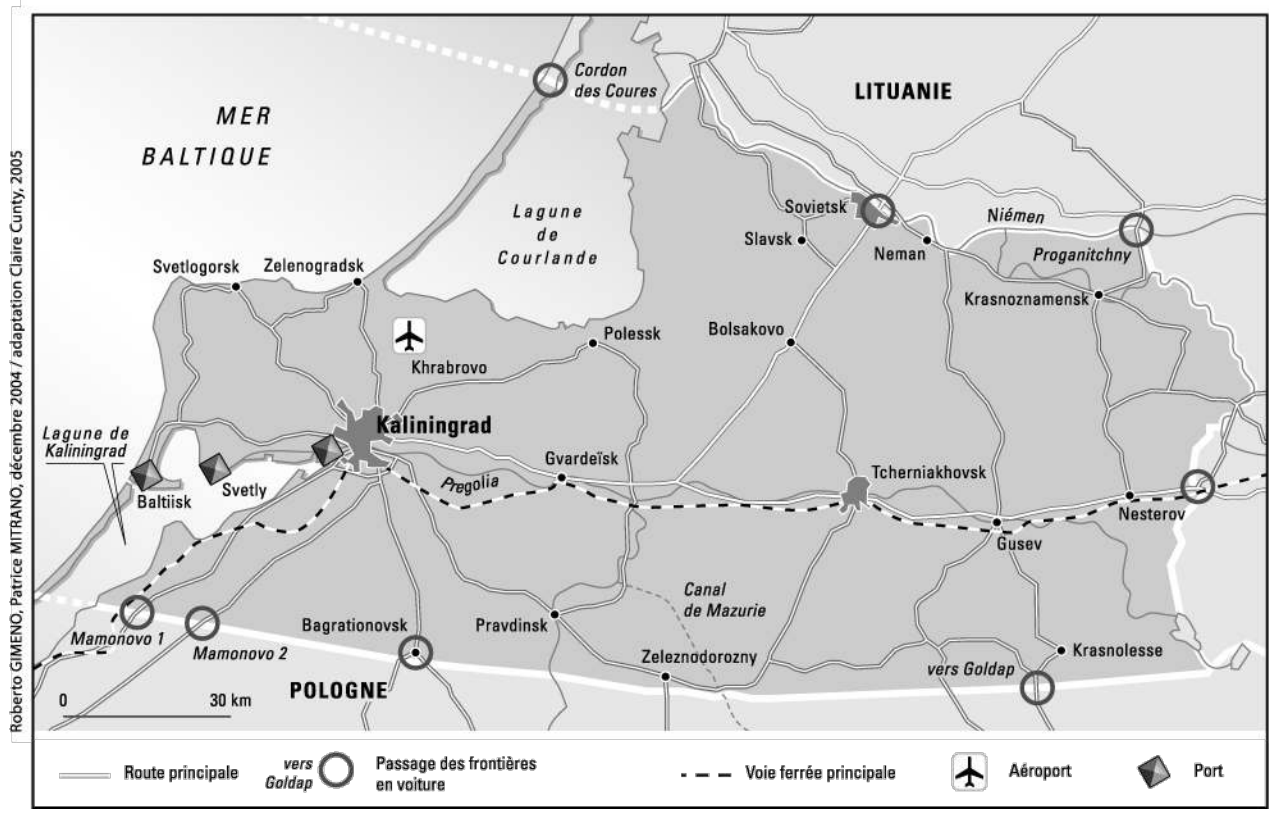

Depuis son aéroport de Khrabrovo, Kaliningrad dessert également plusieurs Etats de la CEI (Biélorussie, Ukraine, Kazakhstan), ainsi que l'Union européenne. L'ouverture de lignes avec l'Europe occidentale vise notamment à favoriser le développement économique régional, en facilitant l'accès de la région aux investisseurs potentiels. Mais elle permet aussi aux habitants de Kaliningrad d'éviter d'avoir à transiter par Moscou pour se rendre en Europe ou ailleurs dans le monde. Après Varsovie (vol quotidien opéré par la compagnie polonaise LOT), la ligne vers Copenhague interrompue fin 2001, suite aux difficultés dans le secteur aérien, a été relancée par une compagnie privée danoise au printemps 2004. Des vols hebdomadaires ou saisonniers existent aussi avec l'Allemagne (Berlin, Hanovre et Munich) depuis 2004 [Tétart, 2004]. Ce réseau aérien international encore modeste, pourrait prendre de l'ampleur, si l'on en croit Aeroflot ${ }^{15}$, qui souhaite faire de Kaliningrad son hub en Europe d'ici fin 2007, en raison de sa position géographique exceptionnelle. En accord avec les autorités régionales et du district du Nord-Ouest, elle est en train de construire un nouveau terminal sur l'aéroport de Khrabrovo et de moderniser les infrastructures existantes, pour les adapter aux exigences internationales et ainsi faire de l'avion le principal mode de transport de la région ${ }^{16}$. Pourtant, la faible accessibilité et la population assez réduite de Kaliningrad laissent aujourd'hui planer le doute sur la possible transformation de Kaliningrad en une plateforme aérienne régionale de la Russie.

Grâce à sa façade maritime, une liaison ferry pour le transport de passagers a pu être mise en service fin 2002 entre le port de Baltiisk et Saint-Pétersbourg, l'autre port russe sur la Baltique. Le temps de traversée est de 32 heures et son prix est comparable à celui d'un billet de train pour le même trajet [Zöllter, 2003]. Or la lenteur de la construction d'aménagements adéquats à Baltiisk - qui reste avant tout un port militaire $-d u$ fait de longues rivalités de pouvoir entre autorités militaires et civiles, n'a pas encore permis à cette route maritime de jouer pour la région de Kaliningrad le même rôle que l'avion. Du moins pour les habitants de Kaliningrad, car au niveau du fret, plusieurs lignes cargos ont été mises en place une à deux fois par semaine vers Kiel, Klaipeda, Hambourg, Saint-Pétersbourg, et Lübeck via Baltiisk [Zöllter, 2003]. 


\section{Le développement économique}

En Prusse-Orientale, qui est avant tout dans les années 1920 une province agricole, ces mesures favorisent l'industrialisation de la région. En 1921 est notamment fondée la compagnie Odin de machines et d'acier, l'industrie de la cellulose est modernisée, si bien qu'elle devient la plus moderne du monde en 1930. Cette industrialisation s'appuie également sur l'extension du port de Königsberg hors du centre ville, sur la rive sud du fleuve Pregel, avec la construction de trois darses en 1924. Celle dédiée aux activités industrielles et commerciales est équipée d'une zone de stockage de $100000 \mathrm{~m}^{2}$ et de 30 grues. Deux silos à céréales hauts de 9 étages sont également mis en place, ainsi qu'une zone franche pour le transit, pour centraliser l'exportation du bois et le blé. En outre, le chenal qui relie sur $42 \mathrm{~km}$ le port de Königsberg à la Baltique est aménagé en 1930, afin de porter sa profondeur de 6,5 à 8 mètres et sa largeur à $70 \mathrm{~m}$, faisant du port de Königsberg, le plus moderne de la Baltique [Albinus, 2002].

Dans la perspective de relancer le commerce régional vers les pays de l'Europe orientale, est créée, à l'initiative du maire de Königsberg, Hans Lohmeyer, la Foire Allemande de l'Est (Ostmesse). Inaugurée en grande pompe en 1920 par le Président du Reich allemand Friedrich Ebert, cette foire est fréquentée jusqu'en 1936 par les agents économiques polonais, soviétiques et scandinaves [Albinus, 2002]. L'idée est de refaire de Königsberg un carrefour de commerce entre Est et Ouest, entre Allemagne et Russie. Jusqu'à la Première Guerre mondiale, la capitale provinciale de Königsberg était au cœur des réseaux de relations autour de la Baltique, prenant de l'espace allemand les flux de commerce et de culture pour les transmettre à l'Empire russe et vice-versa. Elle s'appuyait sur la voie de chemin de fer Berlin-Königsberg - St-Pétersbourg inaugurée en 1857, sur son réseau fluvial (y compris les canaux) et ses ports de Königsberg et Pillau, libres de glaces en hiver.

Enfin, à partir de 1928, un programme fédéral pour l'aménagement de la PrusseOrientale est lancé et doté d'un budget de 75 millions de Reichsmarks et d'un prêt américain de 30 millions de Marks ; puis en 1931, une loi d'aide orientale (Osthilfe) est votée pour la Prusse-Orientale, qui fait suite aux différents programmes pour l'Est mis en œuvre à partir de 1922 sous forme de conversion des dettes agricoles, de subventions à la colonisation et de crédits pour les travaux publics ${ }^{17}$.

A l'instar de Königsberg et de sa Foire annuelle (Ostmesse), les autorités locales de Kaliningrad ont fait le pari de l'ouverture espérant tirer partie de la situation particulière et de la position géographique de la région. Une zone économique spéciale est ainsi lancée en 1993, dont l'objectif est de faire de la région une porte d'entrée pour les échanges avec la Russie, un pont entre l'Union européenne et la Russie. Certains rêvent alors de Kaliningrad comme d'» un nouvel Hong Kong sur la Baltique », c'est-àdire suffisamment autonome de Moscou pour pouvoir se développer économiquement.

Comme en Prusse-Orientale, l'aménagement des infrastructures portuaires fait partie des axes prioritaires de développement privilégiés par Kaliningrad pour pallier l'enclavement. Le chenal du port de Kaliningrad, qui mène du port fluvial à la mer Baltique, est approfondi, pour atteindre 10,5 mètres de profondeur et ainsi en accroître l'accessibilité aux cargos de plus gros tonnages $(40000 \mathrm{t})$ en provenance d'Amérique et d'Asie et aux pétroliers $(30000 \mathrm{t})$. A cette fin, le port est équipé fin 2003 d'une

Revue Géographique de l'Est, vol. 47 / 2 | 2007 
gigantesque grue de déchargement - d'ailleurs fabriquée à Kaliningrad - et la compagnieLUKoil a construit à proximité de Svetly un terminal pétrolier d'une capacité de 1,5 million de tonnes, afin d'exporter par voie d'eau le pétrole qu'elle exploite dans la région. Les autorités portuaires de Kaliningrad comptent désormais sur la construction d'un nouveau port en eaux profondes à Baltiisk, prévue avant fin 2009 pour poursuivre le développement du complexe portuaire de Kaliningrad, deuxième plus grand port de commerce russe sur la Baltique, après Saint-Pétersbourg. Selon Gregory Sebov, Directeur de l'administration maritime du port de Kaliningrad, ce complexe portuaire, libre de glaces toute l'année, à la différence de Saint-Pétersbourg, et plus central dans l'espace régional baltique ${ }^{18}$, est appelé à «jouer, comme par le passé, un rôle de voie de transit entre l'Europe et la Russie $»^{19}$.

Ces réponses immédiates au problème de l'enclavement induit par l'adhésion des pays voisins à l'Union européenne le $1^{\mathrm{er}}$ mai 2004 font partie d'un ensemble plus large de mesures définies par le Programme fédéral spécial de développement économique et social de la région de Kaliningrad pour la période 2002-2010, décidé par décret du gouvernement russe le 7 décembre $2001^{20}$. Ce programme met notamment l'accent sur l'amélioration des infrastructures de transport (routes, rails, ports et air), qui doit permettre le développement de Kaliningrad comme "grand centre de logistique ", afin de garantir des intérêts géostratégiques de la Russie dans l'espace baltique. Or il reste un long chemin à parcourir pour voir ce projet se réaliser, même si, sur les 42 projets prévus dans ce programme, 14 concernent les transports, afin de "faire de la région l'un des plus importants nœuds de transport de la Russie ", pour un coût de 14,7 milliards de Roubles (490 millions d'Euros). Parmi ces priorités, l'approvisionnement énergétique de la région de Kaliningrad représente également un véritable défi pour la région discontinue, puisqu'elle dépend à $90 \%$ d'importations d'énergies primaires (gaz et électricité) en provenance de la Russie centrale et de la Lituanie voisine ${ }^{21}$. Afin d'assurer l'indépendance énergétique de la région, une centrale thermique au gaz a été mise en service fin 2005 et la construction d'une deuxième turbine d'ici 2010 donnera la possibilité d'exporter environ un tiers de l'électricité produite vers l'Union européenne. Une centrale hydraulique datant de l'époque allemande a également été réactivée à Pravdinsk et 21 éoliennes ont été mises en place depuis 1998 dans le cadre d'un projet de coopération avec le Danemark [Krug, 2003].

A travers l'étude de ces quelques mesures prises en Prusse-Orientale dans l'Entre-deuxguerres et quelque 50 ans plus tard à Kaliningrad, force est de constater que les problèmes liés à la discontinuité territoriale (transport, développement économique, etc.) parviennent à être contournés, notamment par l'intervention de l'Etat central, sous la forme d'aides, de subventions ou la mise en œuvre d'un programme de développement ciblé. Si l'enclavement est le plus souvent perçu comme un handicap, c'est que la séparation d'avec le reste du pays est vécue comme une vulnérabilité, une atteinte à la souveraineté et une source d'instabilité. Cette représentation reste somme toute psychologique et largement alimentée - voire instrumentalisée - par des discours nationalistes, puisque l'exemple de la Prusse-Orientale montre que c'est dans la perspective d'inciter une opinion publique allemande à accorder de hautes subventions à la Prusse-Orientale qu'est rédigée en janvier 1930 par les chefs de l'administration territoriale de six anciennes provinces orientales de la Prusse, une note intitulée Détresse des provinces orientales de la Prusseet adressée au président du Reich Hindenburg ${ }^{22}$. 

plus anciens et profonds que la seule situation d'enclavement et d'isolement. Ainsi, l'endettement des entreprises agricoles n'est qu'un vieux problème rendu actuel par l'inflation; sans même évoquer la structure de l'agriculture de Prusse-Orientale qui reste entre les mains de grands propriétaires terriens (Junker). Les difficultés de l'agriculture en Prusse-Orientale, point fort de l'économie locale, ne sont donc pas seulement dues après $1919^{23}$ au renchérissement du prix du transport induit par l'enclavement, puisque ce qui est causé par la nouvelle frontière est supprimé par la réduction des tarifs de fret [Boockmann,1992]. Par ailleurs, avec ou sans séparation, Königsberg reste très éloigné des principaux centres économiques allemands, en particulier des bassins houillers et sidérurgiques de la Ruhr, situés à près de 1000 kilomètres, et de Hambourg, la principale porte d'entrée du commerce transatlantique et colonial, à $650 \mathrm{~km}$ de là.

De la même façon, c'est davantage l'inadaptation de l'économie de Kaliningrad à son nouvel environnement, à savoir la discontinuité territoriale avec le reste de la Russie et à un voisinage plus développé économiquement, et par conséquent plus concurrentiel, qui pourrait être nuisible à l'enclave russe. Or par crainte du «séparatisme » qui menacerait la région, Moscou a eu longtemps tendance à réduire la gestion de l'enclavement à une question politique.

31 Alors que l'enclavement demande avant tout des réponses techniques (y compris économiques), il reste appréhendé dans la majorité des cas, comme un problème politique, comme le montre Kaliningrad et la Prusse-Orientale. Chaque enclave ayant le sentiment d'être un cas à part, unique [Nies, 2003].

\section{De l'instrumentalisation géopolitique du corridor de Dantzig}

32 Dans ce contexte, plus que les conséquences pratiques de la séparation, c'est sa valeur symbolique qui fait l'objet d'une préoccupation principale tant pour l'enclave de Prusse-Orientale que pour celle de Kaliningrad.

\section{A. - -Le corridor de Dantzig au cœur des débats des géographes allemands}

Dès le lendemain de la guerre, la presse allemande, dans de virulentes campagnes, juge intolérable que la Province, la "Prusse des chevaliers Teutoniques», la «Prusse des Hohenzollern qui en fut le premier roi » soit séparée de la mère-patrie [Slawski, 1925]. Elle considère que l'Allemagne doit exiger la révision des frontières établies par le traité de Versailles et par conséquent, demander la réincorporation du littoral polonais à l'Allemagne, un territoire qui la coupe de son histoire, de son berceau. Mais ce sont les géographes allemands qui vont le plus et le mieux montrer l'anomalie de ce découpage. En 1919, Alfred Hettner ${ }^{24}$ est l'un des premiers à dénoncer le Traité de paix de Versailles comme "l'un des pires diktats qui soient» et à considérer comme «impossible que cette catastrophe soit de longue durée». Dans un article intitulé la paix et la géographie politiqueparu dans la Geographische Zeitschrift, il écrit : " on a cassé les reins de la nation allemande ", traduisant par là même le handicap dans lequel 
se trouve désormais le pays amputé d'une partie de son "corps» et donc comme incapable de continuer à se mouvoir, à fonctionner. D'autres géographes comme Walter Geisler considèrent qu'on a violé un "organisme » qui avait toute sa légitimité. Il qualifie donc le corridor polonais de « corps étranger» (fremder Bestandteil), qui en amputant la Prusse-Occidentale isole la Prusse-Orientale du reste de l'Allemagne. Dans un article consacré à la frontière germano-polonaise et publié dans la revue Zeitschrift der Gesellschaft für Erdkunde zu Berlin, le professeur Fritz Jaeger rapporte le caractère unique de la situation ${ }^{25}$ : «C'est le seul cas dans la nouvelle organisation de l'Europe où un Etat fut séparé d'une de ses provinces ».Afin de souligner l'aberration de ce nouveau tracé frontalier pour ses principaux initiateurs (les Français à la Conférence de Paris), Jaeger décrit la nouvelle configuration territoriale, en comparant la Prusse-Orientale à la Suisse et la Pologne à la France :

34 "C'est comme si on avait accordé à la Suisse un large couloir le long de la vallée du Rhône afin de lui assurer un accès à la mer. De ce fait, tout le territoire des Alpes françaises serait séparé de la France et ne pourrait communiquer avec le reste du pays qu'uniquement à travers le couloir ou bien par voie de mer. Par conséquent la ville de Marseille et ses environs ne feraient pas partie du couloir, elle constituerait un Etat autonome libre dont la direction des affaires serait laissée à la Suisse ».

Ces débats géographiques exceptionnels pour l'époque sur le problème des frontières et de la définition même du territoire de l'Etat et de la nation ${ }^{26}$ concourent à la naissance de la Geopolitik ${ }^{27}$. C'est le géographe et ancien officier de l'armée allemande, Karl Haushofer (1869-1946), qui est à l'origine de ce mouvement d'idées. Dans Politische Erdkunde und Geopolitik (Géographie politique et géopolitique) publié en 1925, il définit la géopolitique comme « la conscience géographique de l'Etat » et un moyen de libérer l'Allemagne des entraves du Traité de Versailles [Klein, 1986], à tel point que l'on a pu se demander si cette école n'avait pas été créée pour faire échec à Versailles [Korinman, 1990, p. 167]. De fait, pour les « géopoliticiens » qui se regroupent autour de Karl Haushofer, le combat, ce sont ces nouvelles frontières imposées par le Traité de Versailles. La carte de la Prusse-Orientale les consterne en particulier. En bons géographes, ils considèrent, la région entre les fleuves Oder et Memel comme une entité morphologique. Ainsi, pourquoi mettre la Prusse-Orientale à l'écart de l'Allemagne, si ce n'est pour la détacher progressivement de l'Etat allemand? Une argumentation qui alimente quelque 80 ans plus tard les craintes russes d'aliénation de Kaliningrad, par des mouvements nationalistes (polonais, lituanien et allemand), puis de l'Union européenne elle-même ${ }^{28}$ !

Les géopoliticiens allemands ${ }^{29}$ se souviennent en effet des positions polonaises de 1919, qui dans leurs revendications à un accès à la mer, pour des considérations à la fois économiques et stratégiques, envisagent l'accès le plus large possible; la partie Nord de la Prusse-Orientale autour de Königsberg devant soit être annexée, soit former une union douanière et économique avec la Pologne. Les représentants polonais à la Conférence de Paris - soutenus en cela par la France et des géographes français - se justifient alors, en rappelant qu'il faut «voir la Prusse-Orientale comme le foyer des Junkers réactionnaires et qu'un tel procédé est dans l'intérêt de la démocratisation et de la modernisation de l'Allemagne ». Cette volonté de détacher la Prusse de l'Est du reste de l'Allemagne a aussi une dimension sécuritaire, pour éviter que la Pologne ne soit prise en étau [Oberdörfer, 2003]. 


\section{B. Une configuration géographique à l'origine de tensions}

37 Aussi, dès le milieu des années 1920, les tensions avec la Pologne sont-elles récurrentes. Alors que la politique étrangère de Gustav Stresemann cherche, à partir de 1923, à normaliser les relations de l'Allemagne avec ses voisins occidentaux et à réinsérer le pays dans le jeu des puissances (entrée à la Société des Nations en septembre 1925), la question de ses frontières orientales reste en suspens. Le chef de la diplomatie allemande affirme, en plusieurs occasions, que le tracé de la frontière germanopolonaise est inacceptable pour les Allemands et qu'il ne peut être question, comme pour la frontière occidentale (Alsace-Lorraine) d'un «Locarno de l'Est », qui pourrait empêcher sa révision. Dans ce contexte, la fixation de droits prohibitifs sur l'entrée des produits industriels et agricoles polonais en Allemagne conduit, en 1925, à une " guerre douanière $»^{30}$. Au début des années 1930, les élections présidentielle et législatives se traduisent par un raidissement nationaliste, marqué par une tension régulière avec Varsovie alimentée, côté allemand, par des craintes pour la Prusse-Orientale et Dantzig. On assiste même à une véritable psychose dans l'opinion publique sur la question de la Prusse-Orientale, en particulier dans la province même. En février 1931, la publication d'un roman d'anticipation de Hans Nitram intitulé Les Polonais ont franchi la frontière de Prusse-Orientale, est annoncée sur des affiches au slogan alarmant « Attention! Ici Radio des Marches orientales du Reich! Les troupes polonaises ont cette nuit, franchi la frontière de la Prusse-Orientale ", ce qui provoqua une vive émotion parmi la population et causa plusieurs manifestations contre la Pologne [Montfort, 1933]. La presse provinciale dut éditer des notices explicatives et des rectifications, qui n'eurent que peu d'effet. Selon le Frankfurter Zeitung de mai 1932, cela s'est transformé en véritable psychose anti-polonaise, si bien que «pour le moment à Königsberg, on ne peut pas apporter un tas de sable pour réparer une route, sans que quelques cerveaux plein de sagesse interprètent ce fait comme une mesure de protection contre les bombes incendiaires (...) Et quand on creuse sur une place quelconque pour réparer une canalisation, il naitt tout de suite une rumeur ».

Dès sa création, le corridor de Dantzig suscite donc désapprobation et scepticisme dans l'ensemble de la société civile allemande. Sa légitimité est immédiatement remise en cause, puisque la séparation physique de la Prusse-Orientale d'avec le reste de l'Allemagne rappelle symboliquement l'humiliante défaite et les frontières imposées (le fameux « diktat »), contribuant à la représentation de l'Allemagne comme une nation menacée, sur la voie de la disparition progressive. Cette représentation est souvent reprise dans la propagande anti-Versailles ${ }^{31}$; et ce, d'autant plus facilement que l'unité du pays est très récente, puisqu'elle remonte alors à un peu moins de 50 ans.

\section{Du corridor de Dantzig comme représentation géohistorique et géopolitique russe}

39 Or, ironie de l'histoire, à partir de 1991, la partie Nord de ce même territoire, devenue soviétique après 1945 , retrouve la même configuration d'enclavement, révélant des enjeux identiques et ravivant la représentation géo-historique du corridor de Dantzig. A la différence près que cette fois-ci, le territoire enclavé ne dépend plus d'une « mère patrie » située sur son flanc Ouest, mais sur son Est. Dans le raisonnement russe, le corridor de Dantzig reste le déclencheur de la Seconde Guerre mondiale, pour un motif 
irrédentiste. Avec l'élargissement à l'Est de l'Union européenne et de l'OTAN en 2004, l'enclavement de Kaliningrad par l'Union européenne est perçu par Moscou comme une source de tensions potentielle avec Bruxelles? Voire avec les Etats Baltes, dont la perte par Moscou, après la fin de l'Union soviétique, a été mal acceptée ?

De fait, dans la perception prédominante russe, l'indépendance des pays baltes dans l'Entre Deux Guerres ne constituait qu'une exception due à la faiblesse de la Russie, puisque dans cette représentation, la région appartient depuis toujours à la Russie [Nies (1), 2004]. Face à l'élargissement de l'Union européenne et de l'OTAN, qu'ils ont difficilement accepté, les Russes ne veulent pas, de nouveau, se retrouver dans une position de faiblesse. En se référant à plusieurs reprises au terme " corridor ", que l'on retrouve dans la presse russe, durant la période précédant les négociations entre Bruxelles et Moscou au sujet du transit de et vers Kaliningrad (2000-2002), mais aussi dans la bouche du Président russe Vladimir Poutine lui-même, les dirigeants russes aspirent sans doute à délivrer le message suivant : qu'ils sont en position de force et dans leur bon droit, et donc prêts à affronter les conséquences de la re-création éventuelle par les Européens d'un «nouveau corridor de Dantzig » entravant la libre circulation des citoyens russes, dont la seule Union européenne serait tenue pour responsable. En s'appuyant sur l'histoire et l'image forte de conflit qu'elle contient dans le cas présent, les Russes cherchent à se dédouaner des conséquences négatives que pourrait avoir leur politique vis-à-vis de l'Union européenne concernant Kaliningrad, et ainsi prouver le bien fondé de leur position. Au nom de la libre circulation sans entrave des Russes au sein de leur territoire national, ils veulent éviter à tout prix la mise en place de visas pour le transit ferroviaire et automobile des Russes via la Lituanie.

41 Pourtant, la crainte de voir ressurgir « un nouveau corridor de Dantzig » dans la région est également partagée par les deux voisins de Kaliningrad, la Pologne et la Lituanie. Pour la Pologne, il est en effet hors de question de revivre la situation de l'après Première Guerre mondiale qui avait abouti à sa disparition. Elle a donc catégoriquement refusé tout projet de transit de Kaliningrad vers la Russie, via son territoire et la Biélorussie, envisagé un temps par Moscou. En ce qui concerne la Lituanie, ce sont également des considérations historiques (le viol de son territoire par l'Armée rouge en 1940), moins de 20 ans après son indépendance, qui pousse Vilnius à s'opposer à toute initiative d'un passage extraterritorial à travers le pays. En outre, l'éventuelle mise en place de "trains" par Moscou, via la Lituanie ou la Pologne, évoque aux uns, les pires souvenirs de la déportation des populations juives pendant la Seconde Guerre mondiale, et aux autres, les dispositions mêmes du corridor de Dantzig [Nies, 2003]. Ces deux Etats rejetèrent donc toute proposition de «corridors extraterritoriaux $\|^{32}$, tout comme les Russes qui jugent finalement hérétique de reconstituer des voies terrestres réservées aux seuls autochtones souhaitant se déplacer entre l'enclave et la métropole, comme pendant la Guerre Froide, les autoroutes de transit spécialement désignées et surveillées sur le territoire de la RDA, pour relier Berlin Ouest et la RFA [Chillaud, 2003].

Reste que, pour les habitants de Kaliningrad, mais aussi pour la Russie, la liberté de circulation est perçue autant comme une nécessité qu'un droit. Une nécessité pour l'économie de la région qui dépend des exportations, mais aussi à un niveau psychologique, pour relativiser le sentiment de «vivre dans une souricière » ou " comme des Indiens parqués dans une réserve $»^{33}$. Il s'agit également de permettre aux 
habitants de Kaliningrad de se rendre facilement dans le reste du pays et d'éviter que la Pologne et la Lituanie ne soient plus familières aux écoliers et étudiants de Kaliningrad, qui, selon le Ministère des Affaires étrangères de Russie, ne visiteront plus la Russie, s'il leur faut un visa ${ }^{34}$. Considérées par les Russes comme un droit humain fondamental, les dispositions favorables de libre passage vers la Lituanie sont assimilées à un "acquis ", que les exigences communautaires imposées aux nouveaux Etats membres tendent à remettre en cause, au nom de la libre circulation intérieure à l'espace Schengen. Or, pour Bruxelles, il est inconcevable de troquer les accords de Schengen, en accordant une dérogation pour l'enclave, et pour Moscou, la fin de la libre circulation était perçue comme un risque d'isolement susceptible de favoriser à ses yeux l'autonomisation, voire la sécession de la région par des forces centrifuges (nationalistes allemands, polonais ou lituanien) ou centripètes, comme le Parti républicain balte de Sergéi Pasko, qui prône la création d'une $4^{\mathrm{e}}$ République balte.

Si la position de la Russie se fige alors sur le maintien de la liberté de circulation des Russes de Kaliningrad via la Lituanie, c'est parce que les Russes ne saisissent pas, avant tout par méconnaissance, l'importance de l'acquis communautaire pour l'Union, et donc son intangibilité [Dolan, 2000]. Ainsi, le représentant du Ministère des affaires étrangères de la Fédération de Russie à Kaliningrad, Arthur Kuznetsov n'hésite pas à comparer les pratiques communautaires à celles de "régimes comme ceux de Staline ou Pinochet» [Kuznetsov, 2000]. Pour sa part, Dimitri Rogozine, représentant spécial du Président Poutine pour Kaliningrad, réclame « pas de visa pour voyager à l'intérieur de la Russie $»^{35}$. Quatre cent un députés de la Douma fédérale de Russie allèrent même jusqu'à signer une résolution critiquant de front une « Union Européenne qui préparait les conditions d'une aliénation artificielle de Kaliningrad $»{ }^{36}$ D'autres représentants de l'assemblée russe tentèrent même de semer la zizanie entre la Pologne et la Lituanie, en réclamant la restitution à Varsovie de la capitale lituanienne Vilnius, sous-entendant que la ville sous souveraineté polonaise de 1923 à 1939 avait été injustement rattachée à la Lituanie. Sur place, on s'insurge contre l'entrave faite par la «bureaucratie européenne " à la liberté de mouvement ${ }^{37}$, tout en craignant que les positions maximalistes de Moscou ne soient contreproductives et empêchent des solutions pragmatiques pour le transit des personnes conformes à leur propre intérêt [Timmermann, 2002].

\section{Conclusion : L'enclavement, un enjeu de gouvernance}

$\mathrm{Au}$ fond, la force de la représentation géohistorique du « corridor de Dantzig » a poussé la Russie à ce que le problème du transit via la Lituanie ne devienne pas la source de nouvelles tensions et conflictualités en Europe, ni ne contribue à l'aliénation de Kaliningrad. Le dessein initial du Traité de Versailles d'assurer la paix en Europe et de mettre fin, par un redécoupage des frontières, au balancement entre l'achèvement de la continuité territoriale d'un côté et l'accès à la mer, afin d'acquérir la viabilité économique, de l'autre, s'était avéré vain ; si bien que seule la disparition effective de la Prusse-Orientale en 1945 (par absorption de l'Union soviétique et de la Pologne) sembla apte à apporter une paix durable en Europe, en mettant fin à cette "anomalie géographique ». son intégrité territoriale est déjà menacée en Tchétchénie et que dans la symbolique 
russe, Kaliningrad demeure un "trophée » de la victoire sur le fascisme, lors de la Seconde Guerre mondiale et un tribut aux 20 millions de morts soviétiques. Pourtant, la Russie semble aujourd'hui chercher " par symétrie ", à retrouver les fonctionnalités et la prospérité économique de l'ancienne Prusse-Orientale et de sa capitale mythique, Königsberg. A cet égard, les célébrations des 750 ans de Kaliningrad (ou plutôt de Königsberg) en juillet 2005 ont sans aucun doute contribué à donner une image plus positive de la région ${ }^{38}$, en augmentant son attractivité auprès des acteurs économiques étrangers (avant tout allemands), notamment par la médiatisation de l'événement et la rénovation urbaine alors initiée et qui se poursuit aujourd'hui. Vladimir Poutine n'a-t-il pas annoncé, en septembre 2006, son accord à la reconstruction du Château de Königsberg, symbole du pouvoir des Teutoniques et de la Prusse ? Ce retour sur le passé d'une ville mythique, lieu de naissance du philosophe Kant, ambitionne de rendre son âme européenne à Kaliningrad, et en faire un lieu attractif. Mais il s'accompagne aussi (peut être par crainte du séparatisme) d'une russification de la région avec la construction d'une Cathédrale orthodoxe dans le centre de la capitale, achevée en 2005, et de monuments commémoratifs à la Gloire de la Russie (statues de Pierre le Grand et de la tsarine Elisabeth).

Ce que révèle sans doute le mieux l'approche symétrique de l'enclavement, c'est que les réponses à apporter pour gérer les effets de discontinuité sont avant tout techniques, alors que les Etats souverains sur les enclaves ont tendance à en faire un enjeu purement politique, tant ils sont convaincus de l'unicité de leur situation. L'enclavement est aussi un enjeu de gouvernance - impliquant de fait plusieurs types d'acteurs (société civile, OIG, ONG...) et des critères d'efficacité économique, politique et sécuritaire ${ }^{39}$. Or, tant que la Russie n'aura pas totalement accepté la nature même de Kaliningrad, c'est-à-dire sa position d'enclave, au sein de l'ensemble politicoéconomique qu'est l'Union européenne, la région risque fort de demeurer l'» otage géopolitique $»^{40}$ de Moscou, incapable de contrôler son ouverture et de s'insérer dans les dynamiques politiques et économiques de la région baltique, au détriment de son développement.

\section{BIBLIOGRAPHIE}

ALBINUS R. (2002). - Königsberg Lexikon, Würzburg, Flechsig.

ANCEL J. (1933). - «Géographie politique de la Prusse-Orientale », Conférence faite à la Bibliothèque polonaise de Paris, La Pologne et la Prusse-Orientale, Paris, Société française de librairie Gebethner et Wolff, p. 21-43.

BOoCKMANN H. (1992). - Deutsche Geschichte im Osten Europas, Ostpreußen und Westpreußen, Berlin, Siedler Verlag, $479 \mathrm{p}$.

BINGEN D. et alii (1993). - Das Gebiet Kaliningrad (Königsberg): Bestandsaufnahme und Perspektiven. Deutsche Ansichten I ( $\left.N^{\circ} 21\right)$, Deutsche Ansichten II ( $\left.N^{\circ} 25\right)$. Bundesinstitut für ostwissenschaftliche und internationale Studien, Cologne, respectivement 50 p. et 48 p. 
CHILlAUd M. (2003). - « Kaliningrad et l'ambiguïté de la dynamique des contraires » Géoéconomie, $\mathrm{n}^{\circ} 27$, automne 2003, Paris, revue trimestrielle, pp. 153-176.

DE MONTFORT H. (1933). «L'aspect européen de la question de Prusse-Orientale », Conférence faite à la Bibliothèque polonaise de Paris, La Pologne et la Prusse-Orientale, Paris, Société française de librairie Gebethner et Wolff, p. 119-147.

DE MONTFORT H. (1933). — L'évolution du polonisme en Prusse-Orientale, Paris, Société française de librairie Gebethner et Wolff.

D'HARCOURT R. et alii (1946). - Frontière polono-allemande, La Colombe, Paris.

DOLAN A. (2000). - « Kaliningrad and the European Union : the clash of expectations », in : Baxendale et alii, The EU and Kaliningrad, Federal Trust, Londres, p. 207-213.

GOUNELLE J. (1931). - Le problème de la Basse Vistule, Dantzig et le corridor polonais, Strasbourg, Librairie universitaire d'Alsace.

кокот J. (1958). « Détresse des provinces orientales de la Prusse », Témoignages Allemands, $3^{e}$ cahier, Agence Pologne Occidentale, Poznan.

KORINMAN M. (1990). - Quand l'Allemagne pensait le monde, grandeur et décadence d'une géopolitique, Paris, Fayard.

LOPATA R. (2005). « Kaliningrad, otage géopolitique de la Russie. Un point de vue lituanien. Le courrier des pays de l'Est, n 1048 , mars-avril 2005, la documentation française, Paris, pp. 30-39.

LYNCH D. (2003). - « La Russie face l'Europe », Cahiers de Chaillot n60, mai 2003, Institut d'études de sécurité/Union européenne, Paris.

NIES S. (2004). - Stratégies et sécurité en région balte après le retour des indépendances, 1991-2004, Paris, L'Harmattan.

NIES S. (2004). - Les Etats baltes, une longue dissidence, Armand Colin, Paris.

NIES S. (2003). - Les enclaves : « volcans » éteints ou en activité ? ", La revue internationale et stratégique, $\mathrm{n}^{\circ} 49$, PUF-IRIS, Paris.

NORMANN (von) A. (2002), Nördliches Ostpreußen, Munich, C.H. Beck.

MACHRAY R. (1943). - La Prusse-Orientale et la paix, Londres, F. P Agency Ltd.

OBERDÖRDER L. (2003). - « Konfliktlinien in Ostpreußen am Ende des Ersten Weltkriegs », Osteuropa, Jahrgang 2-3/2003, p. 213-228.

TÉTART F. (2005). - « Kaliningrad, une "île" russe au sein de l'Union européenne élargie », Hérodote $\mathrm{n}^{\circ} 118,3^{\mathrm{e}}$ trimestre 2005, p.175-195.

TÉTART F. (2005). - « Kaliningrad, changer d'image », Questions internationales, $\mathrm{n}^{\circ} 11$, janvierfévrier 2005, Paris, La documentation française, pp. 86-92.

SAMSON I. (2002). - A new look at Kaliningrad, consultable sur le site de l'université Pierre Mendès France de Grenoble, www.upmf-grenoble.fr/gtd/rubrique.php3?id rubrique=2

CASTELLAN G. (1969). - L'Allemagne de Weimar, Paris, Armand Colin.

SLAWSKI S. (1925). - L'accès de la Pologne à la Mer et les intérêts de la Prusse-Orientale, préface de M. Georges LACOUR-GAYET, Paris, éditions Bucard.

ZÖLLTER B.U. (2003). - « Der Ausbau der logistischen Infrastruktur des Gebietes Kaliningrad » in Osteuropa, 2/3-2003, pp. 304-319. 


\section{NOTES}

1. Unité administrative russe équivalente à un département, voire une région.

2. "A free and secure access to sea ". C'est le 13e point des 14 points du président Wilson concernant l'après-guerre en Europe.

3. Il s'agit d'un des arguments donnés dans la « réponse des puissances alliées et associées aux remarques de la délégation allemande sur les conditions de la paix » [Slawski, 1925].

4. Egalement appelée par les Allemands « couloir polonais », (polnisches Korridor en allemand). Cette expression fut utilisée d'abord par la partie polonaise. Or une fois la région cédée à la Pologne, cette désignation s'enracina rapidement en Allemagne, car elle traduisait "dans l'acception allemande l'anomalie de l'état actuel, tout comme l'expression État saisonnier (Saisonstaat en allemand) [utilisée] pour la Pologne ressuscitée ». [Boockmann, 1992].

5. Voir Boockmann, ibid. "In Deutschland wie in Polen meinte man, geopolitische Vorgegebenheiten zu erkennen, die zu sich wiederholenden Konstellation führen - als sei es schon im Mittelalter um den polnischen Zugang zur Ostsee gegangen, als sei schon damals die Landverbindung zwischen dem Reich und Preußen ein politisches Ziel auf deutscher Seite gewesen und darüber hinaus fast so etwas wie ein natürliches Recht, als das umgekehrt auch der polnische Zugang zum Meer galt ».

6. Annexé de facto à l'Union soviétique, ce territoire représentait pour Staline une sorte de « tribut de guerre ", contrepartie des pertes humaines subies par les Soviétiques pendant la guerre (environ 20 millions de morts).

7. Le premier train organisé y arriva le 27 août 1946 en provenance de Briansk. 12024 familles s'installèrent durant cette première année dans la région de Kaliningrad, soit 52906 personnes au total. Les rabatteurs d'État qui parcouraient la Russie centrale, l'Ukraine, la Biélorussie et la Lituanie promettaient de nombreux avantages : l'équivalent de deux ans de salaire, 1000 roubles par membre de la famille et un choix entre un prêt de 3000 roubles ou une vache, sans compter le transfert gratuit jusqu'à Kaliningrad et l'attribution d'une maison.

8. Chapitre XVII du Traité de Versailles intitulé «Prusse-Orientale».

9. Denkschrift des auswärtigen Amts zum Gesetz über das Durchgangsabkomme, Reichstagsdrucksache ${ }^{\circ}$ 2191, cité par Slawski, ibid.

10. La Pologne s'étant engagée à diriger le trafic par la voie la plus courte, ce sont les trois lignes passant par la Poméranie, qui servent au transit allemand : lignes via Lauenbourg-DantzigMarienbourg, via Chojnice-Tczew-Marienbourg et via Schneidemühl-Torun-Dt.Eylau. Voir carte 3.

11. Pour les entrées multiples et avec n'importe quel moyen de transports, il est émis gratuitement pour un période d'un an, auprès des autorités consulaires lituaniennes à partir de juillet 2003. Pour les voyages simples par train en direction ou en provenance de Moscou et SaintPétersbourg, un " document de voyage ferroviaire facilité » (FRTD- Facilitated Rail Travel Document) est émis au moment de l'achat du billet de train, mais il peut être refusé par les autorités lituaniennes.

12. Ancienne dénomination allemande de Tallinn.

13. Le billet d'Aeroflot passe ainsi de 2500 à 900 roubles, soit l'équivalent de 28 Ä pour un allerretour Kaliningrad-Moscou. Devant le succès du programme « Kaliningrad shuttle » qualifié de " social " par Aeroflot, la fréquence des vols passe depuis la saison estivale 2004 de 4 à 5 vols quotidiens, soit 35 vols par semaine. Sur ce programme, voir le site d'Aeroflot :www.aeroflot.ru/ catalog.asp?_no=143\&dno=2989 [10-6-2005].

14. Voir www.strana.ru/print/147346.html [10-6-2005].

15. Selon le communiqué de presse de la compagnie du 17 mars 2004 :www.aeroflot.ru/ catalog.asp?ob_no=143\&dno=2989 [10-5-2004].

16. Selon le représentant du Président russe du district du Nord-Ouest, Ilia Klebanov, ibid. 
17. Ostpreussenprogramm (1922) ; Sofortprogramm (1926), Ostpreussenhilfgesetz (1929); Sofortprogramm (1931).

18. Il est situé à $350 \mathrm{~km}$ de Vilnius, $390 \mathrm{~km}$ de Riga, $400 \mathrm{~km}$ de Varsovie, $550 \mathrm{~km}$ de Minsk, $600 \mathrm{~km}$ de Berlin, Stockholm, $680 \mathrm{~km}$ de Copenhague et $1000 \mathrm{~km}$ de Saint-Pétersbourg.

19. Entretien avec l'auteur en septembre 2003.

20. Le programme ( O federalnoï celevoï programme razvitiya Kaliningradskoï Oblasti na period do 2010 goda ») est disponible dans sa totalité sur le site : www.gov.kaliningrad.ru/ ofederal.php3 [15-6-2003]

21. Voir Königsberger Express, $n^{\circ} 4 / 04$ «Zankapfel Erdgas ». Ce legs de l'époque soviétique représente un défi du fait de la dépendance pour le gaz vis-à-vis des pays de transit (Biélorussie et Lituanie) et du probable raccordement des nouveaux entrants, notamment les pays Baltes, au réseau électrique d'Europe occidentale, sans oublier la fermeture de la centrale nucléaire lituanienne d'Ignalina exigée par Bruxelles entre 2005 et 2009, qui fournit Kaliningrad en électricité. Le risque lié à la dépendance de la région au transit du gazoduc alimentant Kaliningrad via la Biélorussie et la Lituanie a pu être appréhendé par les habitants et autorités de Kaliningrad en février 2004, lorsque Moscou a décidé de ne plus approvisionner en gaz la Biélorussie, qui ne voulait pas accepter les nouveaux tarifs imposés par Moscou. Or ce qui s'avéra être un moyen de pression efficace dans cette « guerre du gaz » entre la Russie et la Biélorussie, fut effectué par Moscou sans se soucier du sort de Kaliningrad.

22. Die Not der preussischen Ostprovinzen, commentée par Jozef Kokot (in biblio).

23. Ses excédents permettaient dès le $\mathrm{XIX}^{\mathrm{e}}$ siècle de fournir d'autres régions du pays. Avec les surplus de céréales, pommes de terres et viandes, 2,5 millions de personnes pouvaient être nourries, soit deux fois que la population de la Prusse-Orientale [Normann, 2002, p. 17].

24. La plupart des exemples concernant les géographes et la géographie politique sont extraits de Korinman [1990, p. 139-170].

25. Cité par Slawski, ibid, comme un exemple de propagande allemande.

26. Voir le préambule d'Yves Lacoste au Dictionnaire de géopolitique, Paris, Flammarion, 1992, pp. 11-13.

27. Puisque contrairement à ceux qui affirment que Friedrich Ratzel est en quelque sorte le fondateur de la géopolitique, c'est seulement après 1918 que débutera avec Haushofer ce que l'on peut appeler, le premier mouvement d'idées géopolitiques. C'est d'ailleurs le thème de la thèse de Michel Korinman intitulée «Quand l'Allemagne pensait le monde éditée en 1990.

28. Voir notamment Bingen (1993) et Tétart (2005).

29. Cf. Leo Wittschell en 1926, dans un article intitulé "Tatsachen und Betrachtungen zur Geopolitik Ostpreußens ", (Faits et observations sur la géopolitique de Prusse-Orientale) in Zeitschrift für Geopolitik n 6 : " l'objectif des Polonais est l'annexion à terme de la PrusseOrientale, à laquelle la Pologne concéderait une forme d'autonomie ».

30. Une situation, qui selon Henri de Montfort (op. cit in biblio) est le fait des nationalistes allemands, par crainte que des relations commerciales trop poussées avec la Pologne ne rendent la Prusse-Orientale dépendante de cette dernière.

31. Par exemple, «La Prusse-Orientale est isolée et condamnée à être noyée dans les flots slaves $»$ in Slawski, op. cit.

32. Cf. Le Monde du 8-3-2002, les propos du Premier ministre polonais Lezcek Miller.

33. Selon les termes de Andréi Vinogradov, député du parti Unité (formation du président Poutine) à la Douma régionale de Kaliningrad, cités par Marie Jego, Le Monde du 21-6-2002, « La Russie refuse de voir Kaliningrad isolée dans l'Europe ».

34. Business in Russia, May-June 2001: "A Russian island in the EU », Natalia Ratiani.

35. Izvestia 20.6.02 : «La Douma protège Kaliningrad ». A titre indicatif, il semble nécessaire de rappeler que les citoyens européens souhaitant se rendre en Russie sont soumis par les autorités russes à l'obtention d'un visa payant, qui nécessite lui-même l'obtention d'une invitation et la 
souscription à une police d'assurances, sans compter que sur place, les voyageurs doivent se faire enregistrer auprès des autorités locales, afin d'obtenir contre paiement un formulaire exigé à la sortie du territoire russe!

36. Rusika Izvestia 20.6.02 «Pas un centimètre de la terre russe ».

37. Le Monde du 21 Juin 2002, «La Russie refuse de voir Kaliningrad isolée de l'Europe ».

38. Cf. Tétart F., « Kaliningrad, redécouvre Königsberg », Le Monde, 3-4 juillet 2005.

39. Voir Susanne Nies, Sands in the works : enclaves challenging Metropolitan States, HDR sous la direction de Bertrand Badie, Paris, IEP Paris, juin 2004, p37-39.

40. Pour reprendre les termes de Raimundas Lopata, Directeur de l'Institut de relations internationales et de science politique à l'Université de Vilnius [Lopata, 2005].

\section{RÉSUMÉS}

Avec la dissolution de l'Union soviétique à la fin de l'année 1991 est apparu sur nos cartes européennes, entre la Pologne et la Lituanie nouvellement indépendante, un petit territoire demeuré sous souveraineté russe, mais désormais séparé du reste de la Russie: l'oblast de Kaliningrad $^{1}$. En prenant pour axe de réflexion ce territoire singulier à deux périodes et - hasard de l'histoire - sous deux souverainetés différentes, l'approche symétrique révèle une configuration répétée de discontinuité territoriale en tant qu'enclave, vis-à-vis de l'Etat central (métropole), puisque jusqu'en 1945, la région de Kaliningrad était la partie Nord de la PrusseOrientale, séparée de l'Allemagne par le «fameux » corridor de Dantzig. L'analyse comparée de Kaliningrad et de son double historique, la Prusse-Orientale, souligne combien l'histoire laisse de traces matérielles sur les territoires, mais aussi dans les représentations géo-historiques, qui sont aussi déterminantes pour modeler et influencer la prise de décision politique. Mais cette analyse régionale rappelle combien l'enjeu de l'enclavement est somme toute plus politique que géographique, tant la gestion de l'enclavement est source de tensions - plus ou moins graves entre l'État souverain sur l'enclave et l'État enclavant.

After the breaking up of the Soviet Union at the end of the year 1991, a small territory still under Russian sovereignty but cut off from the rest of the Russian Federation appeared on the European maps, tucked between the newly independent Lithuania and Poland: the region of Kaliningrad. By analysing this particular territory as an axis at two different periods of time, corresponding - fate's whim - to two different sovereignties, the symmetrical approach reveals a repeated configuration of territorial discontinuity as an enclave vis-à-vis the motherland, since the Russian region of Kaliningrad used to be the Northern part of East Prussia till 1945, which was separated from the rest of Germany by the famous corridor of Dantzig. The comparative analysis of Kaliningrad and of its historical double - East Prussia - shows that history leaves material marks in territories, but also in geo-historical representations, which often play an important role in the political decision-making processes. This region's analysis also demonstrates that enclave governance is a source of more or less severe tensions between the sovereign State on the enclave and the enclave-surrounding State.

Nach der Auflösung der UdSSR Ende 1991 entstand die Enklave Kaliningrad, zwischen dem erneut unabhängigen Staaten Litauen und Polen, und unter russischer Hoheit verbleibend. Wenn man dieses außergewöhnliche Gebiet als Forschungsthema einer "symmetrischen Analyse » 
privilegiert, und das für zwei verschiedene historische Momente, die zufälligerweise zwei verschiedenen Souveränitäten entsprechen, so wird deutlich, dass Kaliningrad wieder und wieder Enklave war. Denn bis 1945 war das Gebiet der nördliche Teil Ostpreußen, das durch den bekannten Danziger Korridor von Deutschland abgetrennt war. Der Vergleich Kaliningrads mit seinem geschichtlichen Doppelgänger, Ostpreußen, zeigt, dass die Geschichte in den Gebieten Spuren hinterlässt, aber auch in geographisch-geschichtlichen Perzeptionen, denen ja meist in Entscheidungsprozessen eine hervorragende Rolle zukommt. Diese regionale Analyse unterstreicht aber auch, wie politisch das Problem des Enklaviertseins ist, insofern die Überwindung der spezifischen Problematik oft Interessenkonflikte und Spannungen zwischen dem über die Enklave souverän bestimmenden Staate und dem um die Enklave liegenden Staat mit sich bringen kann.

INDEX

Mots-clés : symétrie, enclavement, discontinuité, Kaliningrad, Russie, Allemagne

Schlüsselwörter : Symmetrie, Enklaviertsein, Diskontinuität, Kaliningrad, Rußland, Deutschland Keywords : symmetry, enclavement, discontinuity, Kaliningrad, Russia, Germany

\section{AUTEUR}

FRANK TETART

Docteur en géopolitique, frank.tetart@wanadoo.fr 